\title{
The Use of Symbols in Schindler's List
}

\author{
Xiaolin Chen \\ Center for Digitalized Culture and Media, UESTC \\ School of Public Administration, UESTC \\ University of Electronic Science and Technology of China \\ Chengdu, China
}

\begin{abstract}
The basic carriers for expressing emotions in movies are symbols, including linguistic signs and nonlinguistic signs. The Schindler's List is a film that full of symbolic elements. In fact, the film itself has its unique metaphorical meanings and specific symbol system. This symbol system will show the symbolism in the movie, promote the development of the story in a unique ideographic manner, so as to accurately convey the intention of the director. Therefore, the essence of the film can only be delivered by appearance which through the use of the correct interpretation of the deeper levels of spiritual culture and other connotations.
\end{abstract}

Keywords-Schindler's List; non-linguistic symbols; dissemination code; metaphor

\section{INTRODUCTION}

There are two major categories of symbols: the language symbol and the non-linguistic symbol. Metz thinks that a movie is also a language symbol, but unlike the language symbols we already know, it belongs to a special kind of language symbol. It is similar to other language symbols, but no the same. Usually, we analyze language by separate the language into basic units or sense groups. Conventional semantic groups are analyzed by linguistic methods, which can be decomposed into meaningful units such as words, and then separated according to these units and meaning groups to get smaller syllables. In Metz's eyes, the movie achieved the same result by different methods. The film can be broken down into meaningful units such as a signal shot. Each shot can be subdivided again, which contains pictures, audio, and so on. The information in this shot is extremely large compared to ordinary language symbol analysis. Furthermore, the meaning of conventional linguistic signs is formed through social recognition, but the connotation meaning of movie symbols can be instantly created by human beings. Metz accepted Saussure's point of view and incorporated its four cores into the movie. First is the concept of language and speech. Language is a customary expression in our daily life and is widely accepted as a symbolic system. In the case of language, it is a personal ideographic behavior. In other words, our speech can be considered as an activity in which an ideographic system dominates ideographic behavior. The second point, signifier and signified, are arbitrariness established by usage. Symbolism is the signification composed of signifier and signified, while film signage is a series of concerted efforts by the director, Script, Stage, Lighting, Actor, and so on. The third point, combining and aggregating, language is formed by a series of simple word sentences, and word sentences are combined according to simpler simple units, such as phonemes and morphemes. When words can express more different artistic conception through combination and replacement, the mutual combination between symbols is a combination relationship; the symbols that can be replaced with other identical effects are aggregation relations. The fourth point, extension and connotation, is the extension of the signifier and signified to a multi-layered relationship. The bottom-most signification is the signifier of the sign system of the upper level, combined with signified implied previous level is connotation.

\section{SYMBOLIC CODING OF SCHINDLER'S LIST}

\section{A. Symbol and Schindler's List}

In the coding process of Schindler's List, it is a process of visual symbol, which is the main means to express the ideas of the director through the symbols coding. All members of society have a common cultural belief will abide by the way of decode and to understand the meaning behind the code. The cinema code is a symbol system that is regulated by rules and regulations. The coded movie symbols are composed of codes which are understandable and universally applicable. Therefore, the code can be converted among the script, the director and the audience and form a network paradigm that links multiple meanings. In the views of ordinary audiences, it seems that the playback of a movie is nothing more than an actor's performance, intermixing of music, and background scenery. Behind the film production, however, there are several layers of the movie code: the first level is the script, especially the text in the script, and the second level contains not only the first-level but also the visual symbols and auditory symbols. On the third level, all forms of representation are images (visual forms) presented to everyone. This makes it easier for viewers to visualize the effect of the image as a natural reality, while ignoring the symbolic meaning of practice. Like the movies, movies, news, and entertainment programs we see in our daily lives, some of the code that appears on the screen has been largely rooted in the soil of popular social culture, causing them to appear to be not being constructed. Independent and simple visual symbols have natural features in their use. This is not a purely natural representation of visual symbols. On the contrary, in the process of naturalization, it has in fact 
penetrated deeper levels of ideology. The popular belief that naturalized symbols are non-additive symbols which is the most successful manifestation of the "naturalization" of visual symbols. In fact, the effect of implicational signification practice is made up of ideological coding. The movie symbols of Schindler's list are constructed from the following three levels of code:

\section{- First-level code: visual performance}

Makeup, hair style, dress, decoration, expression, performance, gesture, tone, etc.

- Secondary code: technical means

Camera position, light intensity, music, script, etc.

- Third-level code: ideology

Human rights, anti-fascist, etc.

Fiske believes that the actor is a regular performance code, and that the actor's appearance on the screen is processed in accordance with the plot settings and storyline, and is no longer the actual appearance of the actor itself, so the appearance of the actor can be counted as a social one. According to human instincts, when the audience sees an actor in the screen, the audience first concerns their appearance. And whether the appearance of the actor matches the prototype of the story that is depend on the viewer's own understanding of the story and its own culture regular code. The director uses these conventional codes when dealing with the appearance of the character, which makes the actor more sense of substitution and reduces the sense of violation.

In the Schindler's List, Oscar Schindler first appearance was biased, self-centered, proud and arrogant and overobsessed with wealth. Oscar is $193 \mathrm{~cm}$, standing tall and stable temperament, the melancholy and mouthful occasional meaningful smile clearly hides too many stories. The external image of Oscar lies in the process of the development of the plot. At the very beginning, Oscar Schindler's greed and indignation are highlighted. The director uses a lot of the expensive accessories close-up to express, when the conventional code cannot be accurate when the use of social code can show the same effect. Regular codes such as this can be very good at driving story development. The "naturalized" conventional code blessing "social code" in the film. Schindler's List allows viewers to perceive the mimicry environment in the film through the social experience of matching these codes. However, it does not use an objective and fair method or paradigm to perceive the real society. In each movie, characters that are seen as strong and realistic are produced through various kinds of coding and comprehensive processing. Therefore, Fisk said that "reality" is never "original", and what we see is not the original appearance was formed through various processes and combinations at various levels and through the influence of the conventional codes of the media and the influence of social codes to construct a scene that the audience considers "reality".

\section{B. Character Model Code}

The same character has different mentality and character during different periods. The actor's superb acting and wonderful interpretation can bring the audience to in the show. Such a shift is not only completely successful with the acting of the actors, but also from the fact that actors through the social code continue to make their appearance and the characteristics of the characters in the play match and come close to the role. This is also indispensable. This includes first-level codes such as background music for makeup, hairstyles, accessories and secondary angle codes such as shooting angles and lighting positions.

\section{Produce \& Editing Code}

In the process of presenting the visual symbols, the camera creates the various scenarios required for the plot by using the Angle of the machine and the alternating use of close-up lens and long lens, including pictures, angles, motion, tone, color, hue, focal length, distance, Location and lens selection. The camera gives audiences a superior view, a kind of the power of discourse, so that the audiences feel that they open the eye of God, with the identification and anticipation, dominate the dominate the direction of the film story. In our daily life, for example, the display code (Alth): first point, physical contact: touch who, when to touch, transfer the important message of the relationship. Second, in our daily interpersonal communication, space distance determines the degree of intimacy. Intimate distance: less than $45 \mathrm{~cm}$; private distance: $45-120 \mathrm{~cm}$; social distance: $120-$ $360 \mathrm{~cm}$; public distance: more than $360 \mathrm{~cm}$. 5 The close-ups in the movie use the massive of intimate distance to express visual intimacy or danger. Third point, directions: different angle and direction can deliver massage of relationship. Fourth point, nod: for interactive management, especially when talking. One party nods to the other party go on.

\section{THE USE OF LINGUISTIC TYPOLOGY SYMBOLS}

The linguistic typology belongs to one of the many languages of human beings. It is sound but it has no fixed representative meaning. 1 Among them are the voices of nature and the functional sounds humans make, including sobs, giggles, and other common human voices and the sound of the wind and rain, tsunamis, sparrows sing, lions roars, and so on. Music is a unique way of expressing artistic connotation and is a kind of language symbol. An impressive movie will use all sorts of language symbols to transfer the content of the movie. At the same time, it will also promote the development of the plot to stimulate the mood of the audience.

First, Schindler presented with gloomy Sunday, followed by the theme song Remembrance and Theme from Schindler's List slowly interspersed throughout the film, these drastic sounds with sharp and low tone depicts a troubled nation in music way. It makes history heavy and painful to reflect through listening. At this time, the music not only displayed musical notes, movements, and the screen itself, but also describe a numb and insipid portrayal of Schindler's complex myth inner world. Schindler's 
Workforce was played in a strong contrasting picture where the Jews were forced to take care of the family under the supervision of the Nazis and Schindler moved to his new home at the same time. Meanwhile, in the turbulent situation, gunfire broke out in the streets, Nazis are killing the Jews. The entire nation or members can only be dragged down by fate, the Schindler's labor force in the music sounds stubborn, rigid, and blunt. Like the inexorable march of fate crushing the powerless. I Could Have Done More describes Schindler as a war criminal after the end of the war. Composed of clarinet flute strings, the sound is warm and melodious, which is in sharp contrast to the cold weather of the time.

\section{AUDIENCE DECODING}

With the movie's encoding, there is also corresponding audience decoding. From the perspective of communication, the unidirectionality of film communication is strong, and the feedback channels are few. The audience's consumption of film symbols means the completion of the communication process. However, the film symbol system is a complex system composed of visual symbols and auditory symbols, which is a subtle hiding place in the technology. Despite, we know the symbols in the movie, in the same movie, owning to audience has different levels of awareness of the problem, hence, the information and cultural significance is not entirely accepted from the movie to the audience. The extent to which it is not fully acceptable comes from the asymmetric information between the coder and the decoder. Stuart Hall's encoding and decoding theory is that things themselves have no meaning, but representation system through concepts and symbols could make sense. Meaning production relies on the practice of interpretation, and interpretation relies on our active use of the code encoding, the encoding of objects and the translation or decoding of meanings by people on the other end. 2 Hall believes that the interaction between the sender and the receiver builds the text together through coding and decoding. In coding, the communicator can manipulate the text to achieve the purpose of manipulating the audience. In decoding process, the audience will combine their own conditions and situations, such as growth environment education background and social status, etc, with their own unique ways to interpret and then involved in the production of the text. Hall also proposed that the decoding of texts by the audience corresponds to their position and position in the social structure. The upper class of society adopts the mastercontrol reading, the middle layer adopts the negotiated reading, and the lower-level society adopts oppositional reading. 3

\section{A. Hegemonic Reading}

The first master-control reading is also called hegemonic reading. The essence of the film contains the means to realize full transmission, and the audience fully accepts the thoughts of the passer. This ideal situation is the operation of element code to encode information that has been referred to in hegemony. This kind of decoding is to achieve the meaning of the film characterization practice. The practice of film characterization requires the realization of the understanding of the cultural significance of the movie. In terms of cultural significance, the background set by Schindler's List inevitably means the German form. They advocate this complete obedience and worship of the dominant consciousness.

\section{B. Negotiated Reading}

The second type is negotiated reading. The audiences can understand set the predominant order and the encoded hegemonic code. It is clear that decoding contains both compatibility and countermeasures, and then it is possible to decode the individual under the code of the dominant culture. This is neither complete acceptance nor complete negation. Hall believes that the failure of the media in the process of communication originated from the direct conflicts and disagreements between hegemonic code and negotiation code. The audience can develop their own decoding methods to define Got's character: When the cold-hearted, ruthless Got was picking a row of maids standing in front of him, Got actually used his handkerchief to cover her mouth and nose and told them; "I don't want to spread the cold. to you", a little action and blurt out the words to make the murderous Got no longer cold. Then next minute, Got made the decisive order to shoot a Jewish female engineer who opposed him. All in all, under the banner of the leading culture of Schindler's List, the audience can not only follow the filming intentions of the director, but also allow him to see the ugly face of fascists and retain the decoder's independent decoding power. In this way, decode can achieve local and dominant mutual integration.

\section{Oppositional Reading}

The third type is oppositional reading, which is an extreme decoding method. It refers to the audiences' ability to understand the connotations and thoughts that discourse gives to the film, as well as to understand the discourse structure and expression in the dominant cultural order, and to fully see the ideology contained in the context of technical codes. The audience chooses to decode it in the opposite way to the artistic conception and intention created by the writer and director. This kind of decoding method has a kind of meaning to challenge the dominant order. It is most obvious when these people watching and listening to news and current affairs programs on television broadcasts and other media. However, films like Schindler's List have only a few manifestations.

\section{CONCLUSION}

From the point of view of the symbol, the film itself is a symbol system composed of various symbols. In Schindler's List, expect as its excellent language dialogue, nonverbal symbols such as bodies, color, music are bearing and convey more abundant information and the connotation which reflects the deep thoughts and feelings of director. In Schindler's List, all of the symbols play an important role in supporting and complementing. 


\section{REFERENCES}

[1] John Fiske, Introduction to Communication Studies [M], Peking University Press, 2008

[2] Hall S. Representation : cultural representations and signifying practices[J]. Work of Representation, 2002.

[3] Hall S. Representation : cultural representations and signifyingpractices[J]. Work of Representation, 2002. 